\title{
Correction to: Transcatheter valve-in-valve implantation (VinV-TAVR) for failed surgical aortic bioprosthetic valves
}

\author{
Bernhard Wernly ${ }^{1}$ (1) Ann-Katrin Zappe ${ }^{2} \cdot$ Axel Unbehaun $^{3} \cdot$ Jan-Malte Sinning ${ }^{4} \cdot$ Christian Jung $^{5} \cdot$ Won-Keun Kim ${ }^{6}$. \\ Stephan Fichtlscherer ${ }^{7} \cdot$ Michael Lichtenauer $^{1}$ - Uta C. Hoppe ${ }^{1}$. Brunilda Alushi ${ }^{2}$. Frederik Beckhoff ${ }^{2}$. \\ Charlotte Wewetzer $^{2} \cdot$ Marcus Franz $^{8}$. Daniel Kretzschmar ${ }^{8}$. Eliano Navarese ${ }^{9,10,11}$. Ulf Landmesser ${ }^{2}$. \\ Volkmar Falk $3,12,13 \cdot$ Alexander Lauten ${ }^{2,12}$
}

Published online: 4 September 2018

(c) Springer-Verlag GmbH Germany, part of Springer Nature 2018

\section{Correction to: Clinical Research in Cardiology https://doi.org/10.1007/s00392-018-1326-z}

Unfortunately, an error occurred in the original article.

The spelling of the eleventh author's name is "Frederik Beckhoff".

The original article has been corrected.

The original article can be found online at https://doi.org/10.1007/ s00392-018-1326-z.

Alexander Lauten

alexander.lauten@charite.de

1 Clinic of Internal Medicine II, Department of Cardiology, Paracelsus Medical University of Salzburg, Salzburg, Austria

2 Department of Cardiology, Charité-Universitaetsmedizin Berlin, Hindenburgdamm 30, 12203 Berlin, Germany

3 German Heart Center Berlin, Berlin, Germany

4 Department of Medicine II, Heart Center Bonn, University Hospital Bonn, Bonn, Germany

5 Division of Cardiology, Pulmonology, and Vascular Medicine, Medical Faculty, University Duesseldorf, Düsseldorf, Germany

6 Department of Cardiology and Cardiac Surgery, Kerckhoff Heart and Lung Center, Bad Nauheim, Germany
Division of Cardiology, Department of Medicine III, Goethe University Hospital Frankfurt, Frankfurt am Main, Germany

8 Department of Cardiology, Universitätsherzzentrum Thüringen, Friedrich Schiller University Jena, Jena, Germany

9 Inova Center for Thrombosis Research and Drug Development, Inova Heart and Vascular Institute, Fairfax, VA, USA

10 SIRIO MEDICINE Network, Evidence-Based Section, Falls Church, VA, USA

11 Cardiovascular Institute, Ludwik Rydygier Collegium Medicum, Nicolaus Copernicus University, Bydgoszcz, Poland

12 Deutsches Zentrum für Herz-Kreislauf-Forschung (DZHK), Berlin, Germany

13 Charité-Universitätsmedizin, Berlin, Germany 